\title{
Crystal structure solution of an elusive polymorph of Dibenzylsquaramide
}

\author{
Anna Portell, Xavier Alcobé, Latévi M. Lawson Daku, Radovan Černýand Rafel Prohens* \\ Scientific and Technological Centers of the University of Barcelona, \\ C/ Baldiri i Reixac 10,08028 Barcelona, Spain.e-mail: rafel@ccit.ub.edu
}

\footnotetext{
* To whom correspondence should be addressed. Tel. + 3493 4034656. Fax. + 3493 4037206. E.mail: rafel@ccit.ub.edu

${ }^{1}$ Unitat de Polimorfisme i Calorimetria, CCiTUB

${ }^{2}$ Unitat de Difracció de Raigs X, CCiTUB

${ }^{3}$ Laboratory of Crystallography, University of Geneva, Quai Ernest-Ansermet 24, CH-1211 Geneva 4

${ }^{4}$ Dpt. Physical Chemistry, University of Geneva, Quai Ernest-Ansermet 30, CH-1211 Geneva 4
}

\begin{abstract}
:
The crystal structure of the third polymorph of dibenzylsquaramide (Portell, A. et al., 2009), (fig. 1) has been determined from laboratory X-ray powder diffraction data by means of direct space methods using the computing program FOX. (Favre-Nicolin and Černý, 2002) The structure resolution has not been straightforward due to several difficulties on the indexing process and in the space group assignment. The asymmetric unit contains two different conformers, which has implied an additional difficulty during the Rietveld (Rietveld, 1969) refinement. All these issues together with particular structural features of disquaramides are discussed.
\end{abstract}

Key words: dibenzylsquaramide, Crystal structure, X-ray powder diffraction 


\section{INTRODUCTION}

We have recently conducted several studies on disecondary squaramides in the solid state and the existence of a robust head-to-tail supramolecular synthon has been reported. This synthon appears to be unbreakable, unless being the molecule itself covalently modified (Prohens et al., 2012) or particular conformations forced by specific intramolecular interactions (Prohens, Portell and Alcobé, 2012). Polymorphism of dibenzylsquaramide was studied by some of us (Portell et al., 2009), and it was revealed that two room temperature polymorphs, forms B and C, transform into a metastable third form A at the same temperature. Unfortunately, only the crystal structures of forms A and C could be determined. Lattice parameters of form B were suggested but it was not possible to solve its crystal structure. Despite the facts of having a tiny amount of sample and of the preferred orientation problems caused by the habitual needle shape crystals, in this work the use of new high quality glass capillary, good resolution and high statistics laboratory powder XRD data, lead to a successful structure solution using direct space methodology. The results have enabled the discussion of relevant crystallographic features.

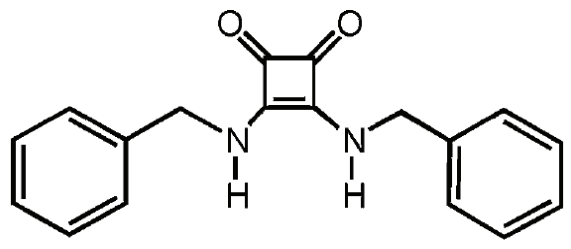

Fig. 1. Dibenzylsquaramide

\section{EXPERIMENTAL:}

\section{A. Synthesis}

The synthesis and structural identity of dibenzylsquaramide were determined spectroscopically $\left({ }^{1} \mathrm{H}-\mathrm{RMN},{ }^{13} \mathrm{C}-\mathrm{NMR}\right)$ and can be found elsewhere (Portell et al., 2009).

\section{B. X-ray diffraction}

Form B was measured in a PANalytical X'Pert PRO MPD powder diffractometer. $\mathrm{Cu} \mathrm{K}_{a}$ radiation, capillary convergent transmission geometry with an elliptic mirror in the incident beam, Soller slits of 0.01 radians and PIXcel detector (active length $3.347^{\circ}$ ). The capillary 
containing the sample was of 0.7 millimeters. Consecutive $2 \theta$ scans from 2 to $70^{\circ}$ were measured and added. The total measuring time was 60 hours.

\section{DFT calculations}

The crystal structure of the form B of dibenzylsquaramide was optimized by density functional theory (DFT) (Hohenberg and Kohn, 1964; Kohn and Sham, 1965) periodic calculations performed within the generalized gradient approximation (GGA) (Perdew et al., 1997) using the Quantum Espresso program package (version 5.0.1). (Giannozzi et al., 1999) The optimization calculations were carried out starting from a Rietveld refined structure. In all calculations, the symmetry was kept fixed to the monoclinic space group $P c$; a $2 \times 10 \times 8$ k-point Monkhorst-Pack grid was used (Monkhorst and Pack, 1976); ultrasoft pseudopotentials were employed ${ }^{1}$ (Vanderbilt, 1990); the wavefunctions were expanded into plane waves up to a kinetic energy cutoff of 60 Ry and a cutoff of 500 Ry was used for the charge density expansion. The optimization calculations were performed both without and with the inclusion of the semiempirical dispersion correction of Grimme (Grimme, 2006) to the GGA functional, as implemented in the Quantum Espresso package. (Barone et al., 2009)

\section{STRUCTURE DETERMINATION AND RIETVELD REFINEMENT OF FORM B:}

The use of high quality, good resolution and high statistics, capillary transmission powder diffraction data has been necessary to solve the crystal structure of form $B$ of dibenzylsquaramide. The right indexation has been obtained using Dicvol04. (Boultif and Louër, 2004) The crystal cell is not orthorhombic as previously reported (Portell et al., 2009) but monoclinic. The cell volume is about half of the orthorhombic previously reported. The final refined cell parameters are: $\mathrm{a}=29.8769(16) \AA, \mathrm{b}=6.0574(2) \AA, \mathrm{c}=8.4662(2) \AA, \beta=$ $97.749(2)^{\circ} ; \mathrm{V}=1518.18(8) \AA^{3}$. Although the space group $P 2_{1} / c$, with $\mathrm{Z}=2$ was in principle possible, the crystal structure was successfully solved with space group $P c$ and $\mathrm{Z}=4$, which implies that there are two molecules in the asymmetric unit. The crystal structure was solved by

${ }^{1}$ We used the ultrasoft pseudopotentials C.pbe-n-rrkjus.UPF, N.pbe-n-rrkjus.UPF, O.pbe-nrrkjus.UPF and H.pbe-rrkjus.UPF from http://www.quantum-espresso.org (last visited October 19, 2012). 
means of direct space methods using the program FOX with the parallel tempering algorithm. In order to accelerate the process during the parallel tempering calculation, the powder pattern was truncated to $2 \theta=35^{\circ}\left(\mathrm{CuK \alpha} \alpha_{1}\right)$. The starting model of 1 was previously optimized with the commercial program SPARTAN and some constraints were introduced to FOX, considering benzyls and cyclobutenes as rigid groups. Several trials of 20 million runs were performed. Subsequently the structure was refined by the Rietveld method with TOPAS 4.14 (Bruker AXS, 2003) and FullProf. (Rodríguez-Carvajal, 1993) Figure 2 depicts the final Rietveld plot. Finally the structure was confirmed by the dispersion-corrected density-functional theory (DFT) calculations.

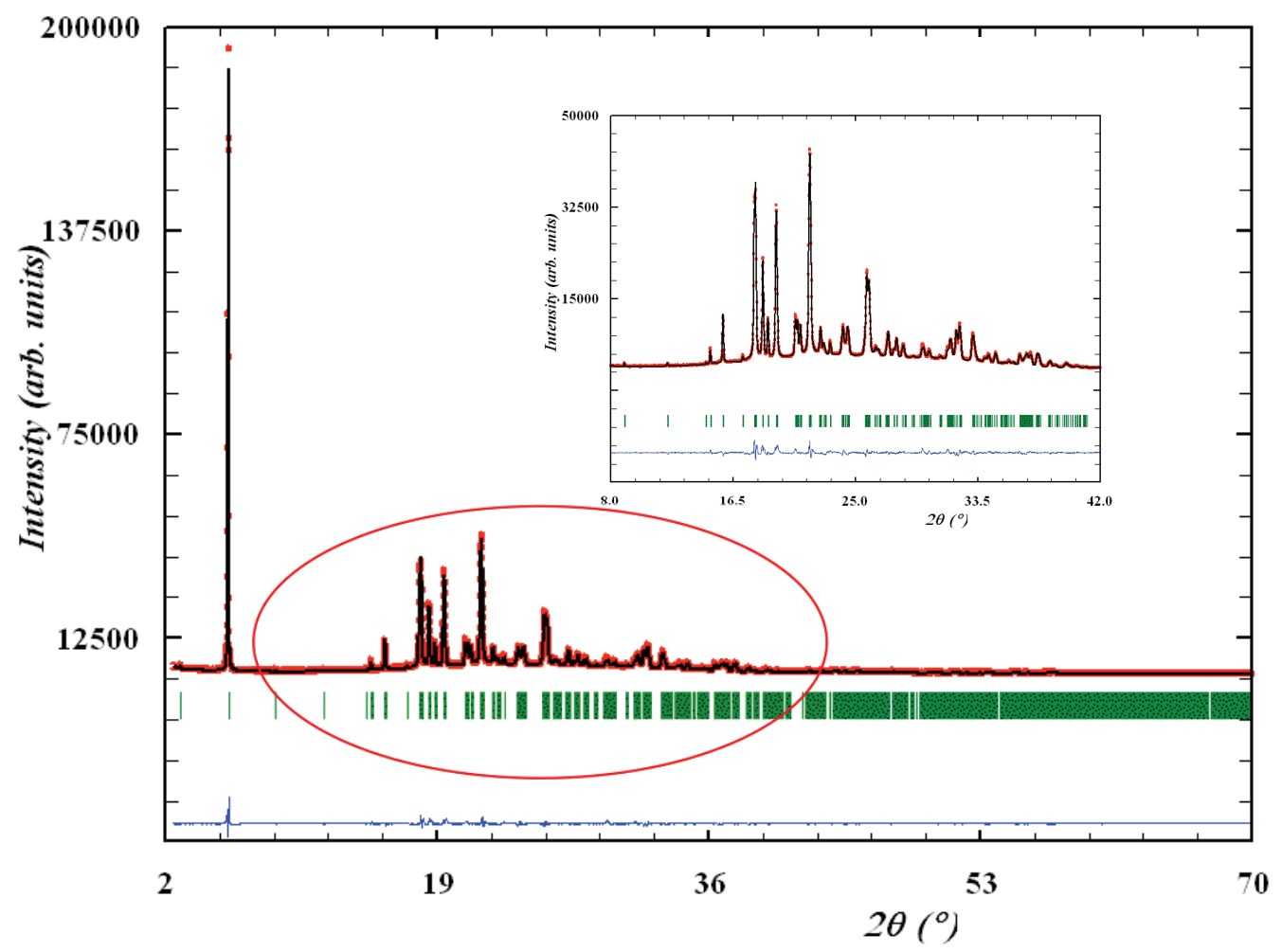

Fig. 2. Final Rietveld plot for the crystal structure refinement of form B of dibenzylsquaramide. The plot shows the experimental powder XRD profile (red marks), the calculated powder XRD profile (black solid line), the difference profile (blue, lower line) and Bragg positions (green lines). Rietveld refinement: $2 \theta$ range: 2.501-69.984º 5191 profile points; 305 refined variables; Rwp $=3.67 \%, \mathrm{Rp}=2.75 \%$ (compared to the Le Bail fit: $\mathrm{Rwp}=2.95 \%, \mathrm{Rp}=1.88 \%$ ).

\section{DISCUSSION}




\section{Description of form B crystal structure:}

Form B crystallizes in $P c$ group with $Z^{\prime}=2$. The two molecules in the asymmetric unit show two different conformations as the result of the aromatic ring rotation (fig. 3).

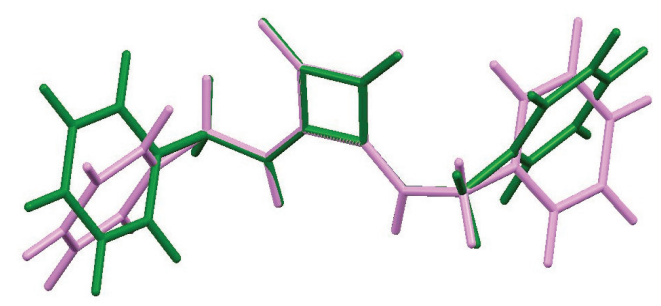

Fig. 3. Overlapped conformers in the asymmetric unit

The main interaction is the expected hydrogen bonded head-to-tail motif in the direction of $a$ axis formed between the two amide hydrogens and the carbonyl oxygens, as we have described recently (Portell et al., 2009; Prohens et al., 2011; Prohens et al., 2012; Prohens, Portell and Alcobé, 2012). Secondary $\pi-\pi$ stacking and $\mathrm{CH}-\pi$ interactions can also be observed in the direction of $b$ and $c$ axis, respectively. The perfectly stacked arrangement of benzene rings was not observed, but a combination of edge-to-face and offset stacked geometry. (Hunter, et al., 2001) Since the asymmetric unit contains two different conformers, there are two types of stacking arrangements. On the one hand, for the first array of molecules, the centroid-centroid distance is $5.27 \AA$, the lateral displacement distance is $4.86 \AA$ and the interplanar distance is $2.05 \AA$, with an angle between the rings of $37.77^{\circ}$. On the other hand, for the second array of molecules, the centroid to centroid distance is $5.18 \AA$, the perpendicular distance between the offset stacked rings is $4.63 \AA$ with a displacement angle of $43.80^{\circ}$ and lateral displacement of $2.3 \AA$. In both cases, the offset stacked molecules form a zig-zag pattern (fig. 4). 


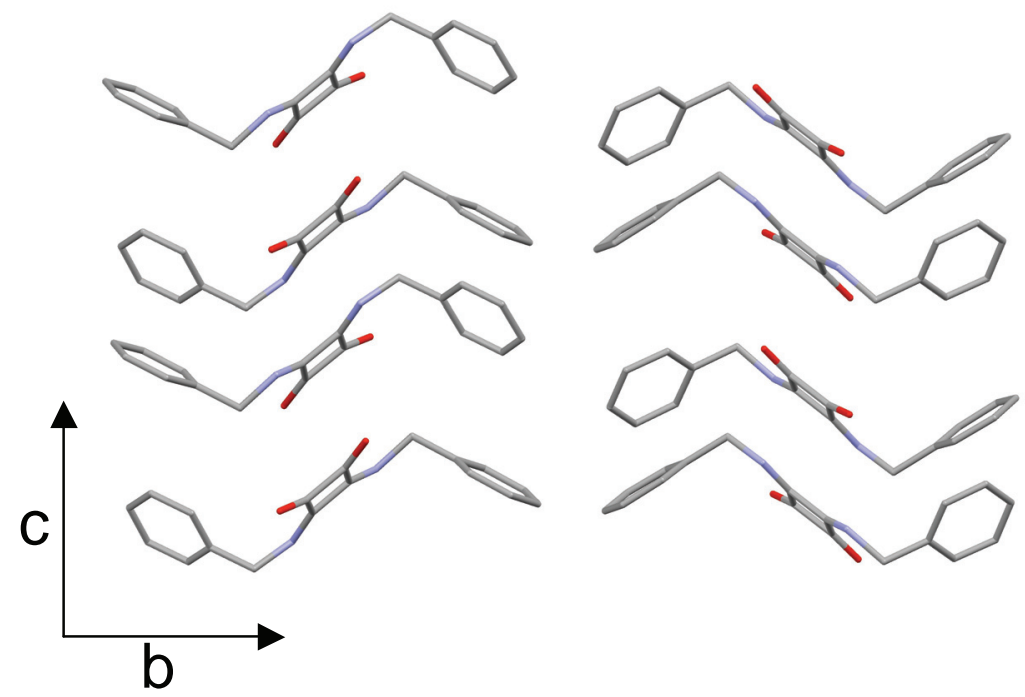

Fig. 4. Crystal structure of form B showing aromatic interactions

Once the structure was refined by the Rietved method, a verification was performed by means of periodic DFT. Generalized-gradient-approximations (GGA), like the PBE functional (Perdew et al., 1997) used in a first stage, tends to give cell parameters which are slightly overestimated. In the present case, however, the optimized "c" parameter is far too large: $\mathrm{c}=9.7066 \AA$, (and a = $30.6321 \AA, b=6.0651 \AA, \beta=97.722^{\circ}$ ). Given that the "c" direction is associated with the stacking of the molecules, the large overstimation of this cell parameter can be ascribed to the difficulties met with most DFT functionals for the accurate description of the van der Waals interactions, which rule the stacking of the molecules. This is confirmed by the results obtained with the dispersion-corrected PBE functional: $\mathrm{a}=29.4422 \AA, \mathrm{b}=5.9643 \AA$, $\mathrm{c}=7.9376 \AA$, $\beta=96.848^{\circ}$; in much closer agreement with experiment.

The fingerprint plots (Spackman and McKinnon, 2002) of the two different conformers in the asymmetric unit show the same hydrogen bonding motif but subtle differences of packing, with $d e$ (left) and $d i$ (right) mapped in colour (in both cases red represents the closest contacts, and blue the most distant contacts) (fig. 5). 

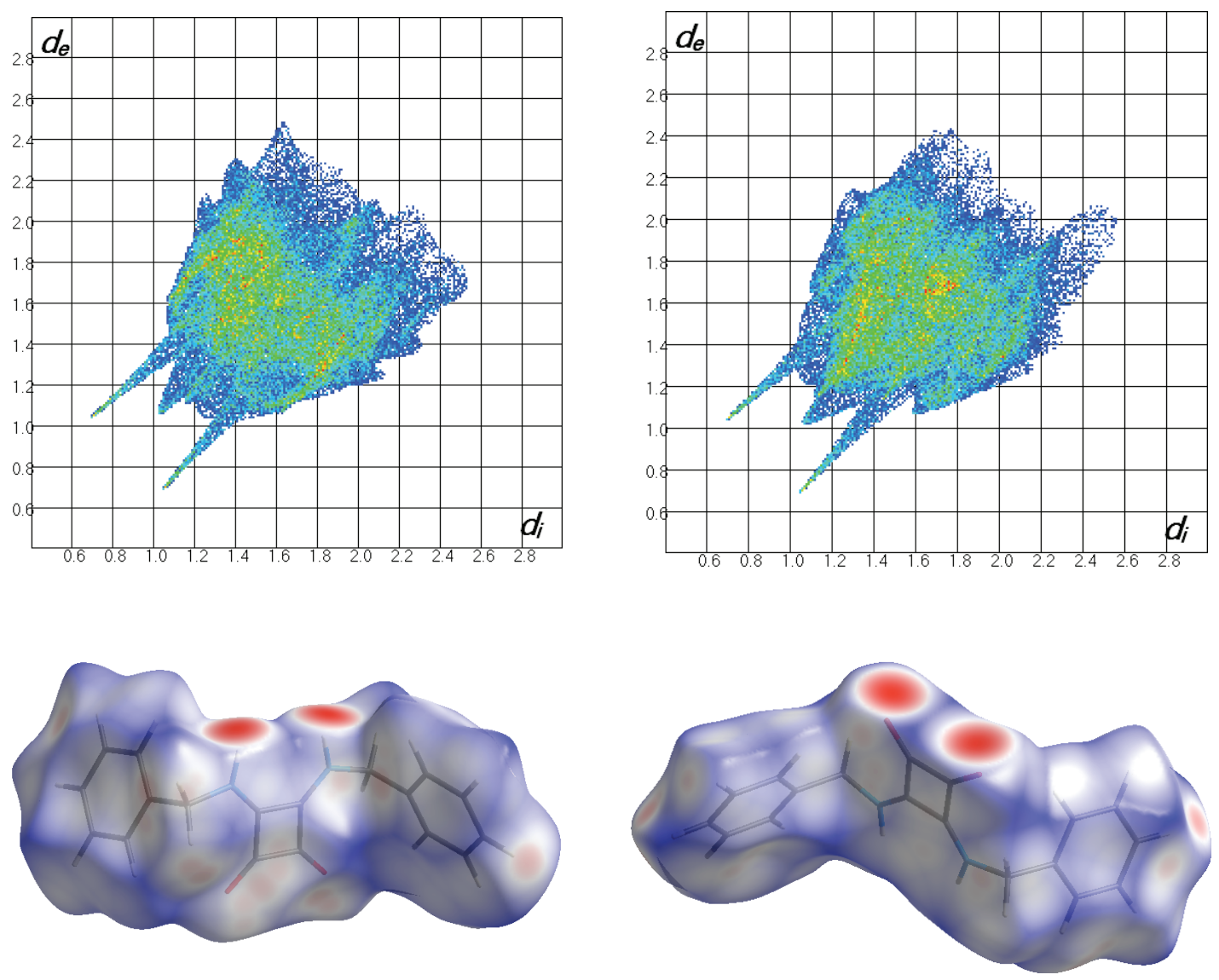

Fig. 5. Hirshfeld surfaces (McKinnon, Spackman and Mitchell, 2004; Wolff et al., 2007) (below) and fingerprint plots (above) of both conformers.

In principle, self-assembled chains of disecondary squaramides can be oriented in three different manners: parallel, antiparallel, and unparallel alignments with implications in the crystal polarity which can produce Non-Linear Optical (NLO) materials (fig. 6). Since the molecular dipole moment of dibenzylsquaramide is high ${ }^{2}$, the most likely alignment expected is the antiparallel

${ }^{2}$ The calculated dipole moment of dibenzylsquaramide is $8.15 \mathrm{D}$ calculated through DFT using the B3LYP/6-31G* functional. 
one, which minimizes the net dipole moment in the crystal. (Glaser, Knotts and Wu, 2003) Form B shows an antiparallel alignment, as previously reported for form C, whereas form A crystallizes in an unparallel configuration, with an angle of $144^{\circ}$ between the dipole moment vectors of slightly antiparallel chains.
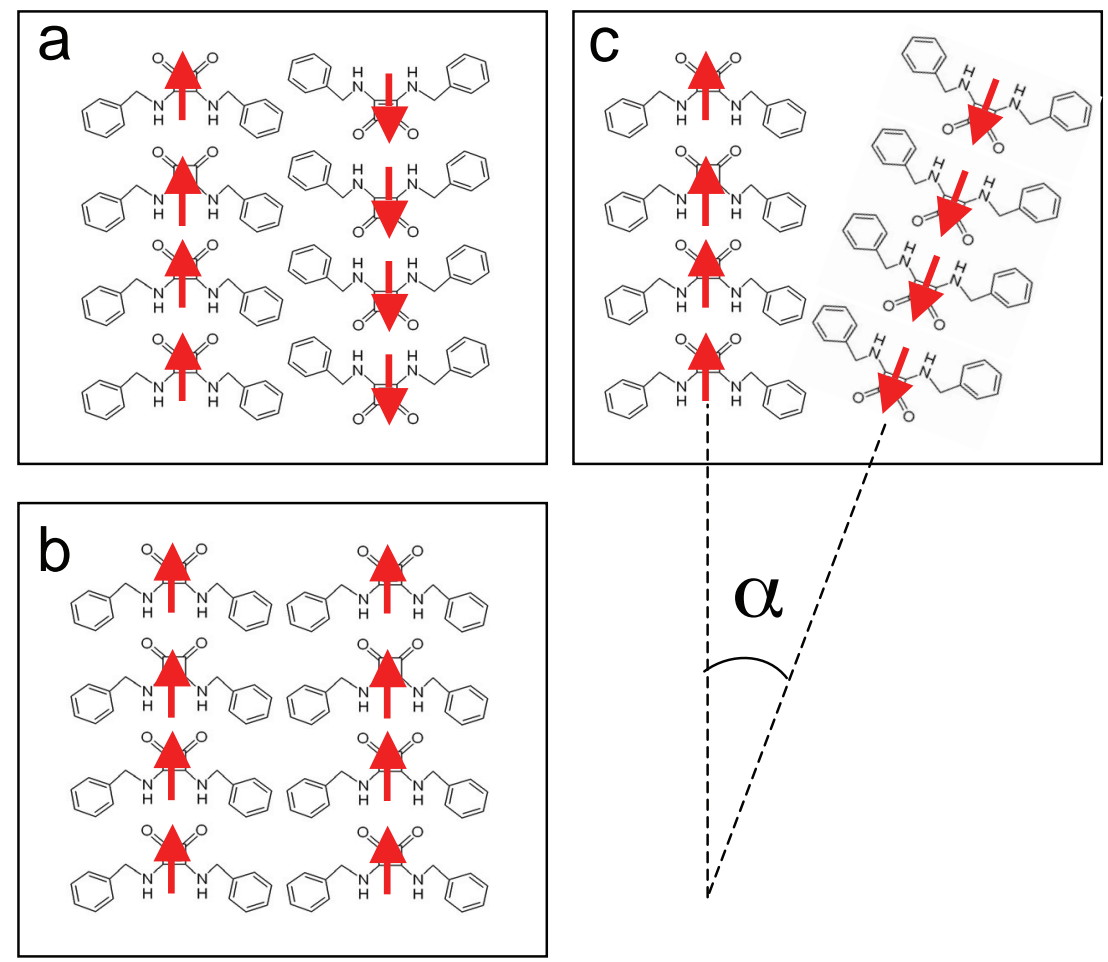

Fig. 6. Different possible combinations of dipole moment alignment; (a) antiparallel, (b) parallel and (c) unparallel, showing the angle between dipole moment vectors.

\section{Conclusions:}

Despite the difficulties met during the indexing process, the crystal structure of an elusive polymorph of dibenzylsquaramide has been solved from good quality laboratory PXRD data. Further studies are being conducted to solve a fourth high temperature polymorph of dibenzylsquaramide, which could convert it in one of the scarce polymorphic compounds with four determined crystal structures. We are currently exploring the NLO properties of form A, which presents a net dipolar moment in the crystal. 
CCDC 914675 contains the supplementary crystallographic data for this paper. These data can be obtained free of charge via www.ccdc.cam.ac.uk/data_request/cif or by emailing data_request@ccdc.cam.ac.uk, or by contacting The Cambridge Crystallographic Data Centre, 12, Union Road, Cambridge CB2 1EZ, UK; fax: +44 1223336033.

\section{Acknowledgements:}

This work was supported by a grant from the Swiss National Supercomputing Centre (CSCS) under project ID s103 and by a grant from the Center for Advanced Modeling Science (CADMOS) under project ID CTESIM. The financial support for CADMOS and for the Blue Gene/P system is provided by Geneva Canton, Vaud Canton, fondation of Hans Wilsdorf, fondation of Louis-Jeantet, the University of Geneva, the University of Lausanne, and the Ecole Polytechnique Fédérale de Lausanne.

Barone, V., Casarin, M., Forrer, D., Pavone, M., Sambi, M. and Vittadini, A., (2009).

"Role and effective treatment of dispersive forces in materials: Polyethylene and graphite crystals as test cases," J. Comput. Chem. 30, 934-939.

Boultif, A. and Louër, D. (2004). "Powder pattern indexing with the dichotomy method," J. Appl. Crystallogr. 37, 724-731.

Bruker AXS. (2003). TOPAS V2.1: General Profile and Structure Analysis Software for Powder Diffraction Data. User's Manual (Bruker AXS, Karlsruhe, Germany).

Favre-Nicolin, V. and Černý, R. (2002).’FOX, 'free objects for crystallography': a modular approach to ab initio structure determination from powder diffraction," J. Appl. Crystallogr. 35, 734-743.

Giannozzi P., Baroni, S., Bonini, N., Calandra, M., Car, R., Cavazzoni, C., Ceresoli, D.

Chiarotti, G. L., Cococcioni, M., Dabo, I., Dal Corso, A., de Gironcoli, S., Fabris, S. Fratesi, G., Gebauer, R., Gerstmann, U., Gougoussis, C., Kokalj, A., Lazzeri, M., Martin-Samos, L., Marzari, N., Mauri, F., Mazzarello, R., Paolini, S., Pasquarello, A., Paulatto, L., Sbraccia, C., Scandolo, S., Sclauzero, G., Seitsonen, A. P, Smogunov, A., Umari, P. and Wentzcovitch R. M. (2009). "QUANTUM ESPRESSO: a modular and open-source software project for quantum simulations of materials,” J. Phys. Condens. Matter, 21, 395502. 
Glaser, R., Knotts, N. and Wu, H. (2003). "Polar Order in Crystalline Molecular Organic Materials by Rational Design,” Chemtracts Org. Chem. 16, 643- 652.

Grimme, S. (2006). "Semiempirical GGA-type density functional constructed with a long-range dispersion correction," J. Comput. Chem. 27, 1787-1799.

Hohenberg, P. and Kohn, W. (1964). "Inhomogeneous Electron Gas," Phys. Rev., 136, B864-B871.

Hunter, A. C., Lawson, K. R., Perkins J and Urch C. J. (2001). "Aromatic interactions," J. Chem. Soc., Perkin Trans. 2, 651-669.

Kohn, W. and Sham, L. J. (1965) "Self-Consistent Equations Including Exchange and Correlation Effects," Phys. Rev. 140, A1133-A1138.

McKinnon, J. J., Spackman M. A., Mitchell, A. S. (2004). “Novel tools for visualizing and exploring intermolecular interactions in molecular crystals," Acta Crystallogr., Sect. B : Struct. Sci. 60, 627-668.

Monkhorst, H. J. and Pack, J. D. (1976). “Special points for Brillouin-zone integrations," Phys. Rev. B 13, 5188-5192.

Perdew, J. P., Burke, K. and Ernzerhof, M. (1997). "Generalized Gradient Approximation Made Simple,” Phys. Rev. Lett. 78, 3865-3868.

Portell, A., Barbas, R., Braga, D., Polito, M., Puigjaner, C. and Prohens, R. (2009). "New polymorphic hydrogen bonding donor-acceptor system with two temperature coincident solid-solid transitions," CrystEngComm 11, 52-54.

Prohens, R.; Portell, A. and Alcobé, X. (2012). "Effect of Preorganization on the Polymorphism and Cocrystallization of a Squaramide Compound," Cryst. Growth Des. 12, 4548-4553.

Prohens, R., Portell, A., Puigjaner, C., Barbas, R., Alcobe, X., Font-Bardia, M. and Tomas, S. (2012). "Cooperative induction in double H-bonding donor/acceptor compounds: Chains vs. ribbons," CrystEngComm 14, 5745-5748.

Prohens, R., Portell, A., Puigjaner, C., Tomas, S, Fujii, K., Harris, K. D. M., Alcobe, X., Font-Bardia, M. and Barbas, R. (2011). "Cooperativity in Solid-State Squaramides," Cryst. Growth Des. 11(9), 3725-3730.

Rietveld, H. M. (1969). “A profile refinement method for nuclear and magnetic structures," J. Appl. Crystallogr. 2 (2), 65-71.

Rodríguez-Carvajal, J. (1993). "Recent advances in magnetic structure determination by neutron powder diffraction," Physica B, 192, 55-69.

Spackman, M. A. and McKinnon, J. J. (2002). "Fingerprinting intermolecular 
interactions in molecular crystals," CrystEngComm 4 (66), 378-392.

Vanderbilt, D. (1990). "Soft Self-Consistent Pseudopotentials in a Generalized Eigenvalue Formalism," Phys. Rev. B 41, 7892-7895.

Wolff, S. K., Grimwood, D. J., McKinnon, J. J., Jayatilaka, D. and Spackman, M. A.

(2007). Illustrations with computer package CrystalExplorer v2.1. (Computer Software), University of Western Australia, Perth, Australia.

Xu, X. and Goddard, W. A. (2004). "The extended Perdew-Burke-Ernzerhof functional

with improved accuracy for thermodynamic and electronic properties of molecular systems," J.

Chem. Phys. 121, 4068. 